\title{
Involvement of GABAergic mechanisms of the dorsal periaqueductal gray and inferior colliculus on unconditioned fear
}

\author{
Adriano Edgar Reimer, Amanda Ribeiro de Oliveira and Marcus Lira Brandão \\ Universidade de São Paulo, Ribeirão Preto, Brazil
}

\begin{abstract}
The fact that the dorsal periaqueductal gray (dPAG) and inferior colliculus (IC), together with superior colliculus, medial hypothalamus and amygdala, constitute the brain aversion system has been well-established. Stepwise increases in the intensity of electrical stimulation of dPAG or IC cause freezing and escape responses, which are followed by a freezing behavior that lasts after the interruption of the stimulation. Freezing and escape are unconditioned defensive behaviors derived from the stimulation of the output centers for the defense reaction, whereas the post-stimulation freezing is the behavioral counterpart of the processing of aversive information. Although GABA-A mechanisms of the midbrain tectum exert a tonic inhibitory influence on the neural substrates of unconditioned fear, their influence on the processing of aversive information is not completely understood. Thus, the present study examines the effects of injections of the GABA-A receptor agonist muscimol ( 1 and $2 \mathrm{nmol} / 0.2 \mu \mathrm{L})$ or the glutamic acid decarboxylase blocker semicarbazide $(5$ and $7.5 \mu \mathrm{g} / 0.2 \mu \mathrm{L})$ into dPAG or IC of Wistar rats on freezing and escape thresholds determined by electrical stimulation of these same structures and on post-stimulation freezing. Intra-dPAG injections of muscimol increased and semicarbazide decreased the freezing and escape thresholds of electrical stimulation of the dPAG. Only semicarbazide enhanced the dPAG post-stimulation freezing. Intra-IC injections of muscimol significantly increased aversive thresholds, while having no effect on IC post-stimulation freezing. Intra-IC injections of semicarbazide had no significant effects. These findings suggest that GABAergic mechanisms are important regulators of the expression of unconditioned fear in IPAG and IC, whereas only in dPAG GABA appears to play a role on the sensory gating towards aversive information during post-stimulation freezing. Keywords: muscimol, semicarbazide, dorsal periaqueductal gray, inferior colliculus, aversive thresholds, post-stimulation freezing.
\end{abstract}

Received 1 December 2008; received in revised form 9 April 2009; accepted 9 April 2009. Available online 29 June 2009

\section{Introduction}

The fact that the dorsal periaqueductal gray (dPAG) and inferior colliculus (IC), together with the superior colliculus, medial hypothalamus and amygdala, are structures involved in the processing of aversive information and constitute the brain aversion system has been well-established (Brandão, Anseloni, Pandóssio, De Araújo, \& Castilho, 1999; Brandão, Tomaz, LeãoBorges, Coimbra, \& Bagri, 1988; Brandão et al., 2005). Indeed, stepwise increases in the intensity of electrical stimulation of dPAG or IC in laboratory animals have

Adriano Edgar Reimer, Amanda Ribeiro de Oliveira and Marcus Lira Brandão - Laboratório de Psicobiologia e Instituto de Neurociências e Comportamento - INeC, Faculdade de Filosofia, Ciências e Letras da Universidade de São Paulo, Ribeirão Preto, SP, Brazil. Corresponding regarding this article should be directed to Marcus Lira Brandão, Laboratório de Psicobiologia, Faculdade de Filosofia, Ciências e Letras da Universidade de São Paulo, Ribeirão Preto, Avenida Bandeirantes 3900, SP, Brazil, 14040-901. Phone (+55 16 3602-4830), E-mail: mbrandao@usp.br. been shown to induce elevations in heart rate and blood pressure, hyperventilation and defensive responses such as arousal, freezing, and flight (Brandão et al., 1988, 1999; Brandão, Melo, \& Cardoso, 1993; Troncoso, Osaki, Masson, Borelli, \& Brandão, 2003). Furthermore, it has been shown that the interruption of the electrical stimulation of the dPAG at the escape threshold gives way to another kind of behavior, the so-called dPAG post-stimulation freezing (Brandão, Zanoveli, RuizMartinez, Oliveira, \& Landeira-Fernandez, 2008; Martinez, Oliveira, \& Brandão, 2006, 2007; Vianna, Graeff, Brandão, \& Landeira-Fernandez, 2001a; Vianna, Landeira-Fernandez, \& Brandão, 2001b). Freezing and escape are unconditioned defensive behaviors derived from the stimulation of the output centers for the defense reaction, whereas the post-stimulation freezing is the behavioral counterpart of the processing of aversive information (Brandão et al., 2008). These defense strategies mimic fearful behavior elicited by environmental challenges and have been used as an animal model of panic attacks (Graeff, 1994; Graeff \& Del-Ben, 2008; Graeff, Silveira, Nogueira, Audi, \& Oliveira, 1993). 
GABA-mediated processes seem to have important physiological roles in both $\mathrm{dPAG}$ and IC, since immunohistochemical studies have shown that these structures contain high concentrations of GABA and its synthesizing enzyme, the glutamic acid decarboxylase (GAD) (Thompson \& McGeer, 1985). Injections into the IC or dPAG of drugs that block the GABA postsynaptic receptors, such as bicuculline, or drugs that inhibit GAD, such as semicarbazide, produce defense reactions. In contrast, injections of the GABA-A agonist muscimol have opposite effects, i.e. inhibitory effects on this defense reaction (Brandão et al., 1988, 1993, 2005; Pandossio \& Brandão, 1999). Because of this, it has been suggested that GABA-A mechanisms exert a tonic inhibitory influence on the neural substrates of aversion in the midbrain tectum (Brandão et al., 2005). Although GABA-A mechanisms of the midbrain tectum exert a tonic inhibitory influence on the neural substrates of unconditioned fear, their influence on the processing of aversive information is not completely understood.

Despite the fact that DPAG and IC are related to the generation and expression of unconditioned fear, their neural substrates of aversion appear to serve two different functions. For instance, the escape behavior generated by the dPAG stimulation is explosive and poorly coordinated, whereas the behavior generated in response to stimulation of the IC is coordinated and less intense (Brandão et al., 1988). Also, electrical stimulation of the inferior colliculus at the escape threshold produces a long-lasting increase in the levels of corticofrontal dopamine, while no significant changes are observed following $\mathrm{dPAG}$ electrical stimulation (Cuadra, Zurita, Macedo, Molina, \& Brandão, 2000). However, the regulation by GABAergic mechanisms of the aversive states induced by electrical stimulation of the APAG and IC is not completely understood.

Panic attacks are sudden surges of intense fear, or terror, accompanied by major neurovegetative changes, such as hypertension, difficulty in deep breathing, urges to void the bladder and increased peristalsis. Panic disorder is characterized by recurrent panic attacks, either unexpected or associated with particular situations, and persistent concern about having another attack or worry about the implications and consequences of the panic attacks (Graeff, 2002, 2004). In order to improve our knowledge on the neurobiology of anxiety disorders, research to identify the neural substrates of the $\mathrm{APAG}$ and IC involved in different types of fear and the underlying neurochemical mechanisms is currently under vigorous investigation. The procedure of electrical stimulation of the dPAG has been employed as a useful animal model of panic disorder. In this respect, freezing and flight responses induced by electrical stimulation of $\mathrm{dPAG}$ and the dPAG post-stimulation freezing have been related to distinct behavioral defensive systems with different biological functions (Brandão et al., 2008; Martinez et al., 2006, 2007).
The combined use of the dPAG stimulation procedure and drug injections into the dPAG itself can be a useful tool in understanding the differences in panic attacks and panic disorder and the effects of anxiolytic medications having distinct mechanisms of action. In this direction, the present study examined the regulatory role of GABAergic mechanisms of $\mathrm{APAG}$ and IC in freezing and escape responses elicited by aversive stimulation of these structures and in post-stimulation freezing.

\section{Materials and Methods}

\section{Subjects}

One-hundred thirty-five naive male Wistar rats from the animal house of the Campus of Ribeirão Preto of the University of São Paulo weighing 250-300 g were used. In order to acclimatize these animals to ambient laboratory conditions, they were transferred to a laboratory animal house adjacent to the test room $72 \mathrm{~h}$ before beginning the experimental trials. The animals were housed in groups of five in a plastic box (33 x $17 \times 40$ $\mathrm{cm})$. The rats were maintained on a $12 \mathrm{~h}$ lightdark cycle (lights on at 7:00 a.m.) with room temperature at $23-25^{\circ} \mathrm{C}$ and allowed free access to food and water in their home cages throughout the experiment. All protocols of this study were approved by the ethics committee of University of São Paulo (06.1.123.8.53.9) and were followed according to the rules for animal experimentation of the SBNeC (Brazilian Society of Neuroscience and Behavior) which are based on the United States National Institutes of Health Guide for Care and Use of Laboratory Animals

\section{Surgery}

The animals were anesthetized with tribromoethanol (250 mg/Kg, i.p.) and fixed in a stereotaxic frame (David Kopf, CA, USA). The upper incisor bar was set at $3.0 \mathrm{~mm}$ below the interaural line, such that the skull was made horizontal between bregma and lambda. A chemitrode made of a stainless steel guide cannula (o.d. $0.6 \mathrm{~mm}$, i.d. $0.4 \mathrm{~mm}$ ) glued to a brain electrode made of stainless steel wire $(160 \mu \mathrm{m}$ in diameter) insulated except at the tip, was unilaterally aimed at the dPAG or IC. Taking lambda as the reference point, the coordinates used were: $\mathrm{dPAG}$ (antero-posterior: $+0.1 \mathrm{~mm}$, medio-lateral: +1.9 $\mathrm{mm}$; dorso-ventral: $-4.3 \mathrm{~mm}$, with a $16^{\circ}$ angle) and IC (antero-posterior: - $0.9 \mathrm{~mm}$, medio-lateral: +1.7 $\mathrm{mm}$; dorso-ventral: $-4.5 \mathrm{~mm}$ ). The chemitrode was fixed to the skull by means of acrylic resin and two stainless steel screws. At the end of the surgery, each guide-cannula was sealed with a stainless steel wire to protect it from congestion. 


\section{Drugs}

The following drugs were used: muscimol (MUSC; RBI, MA, USA) - a GABA-A receptor agonist - and semicarbazide (SMC; Sigma, MO, USA) - an inhibitor of the GABA synthesizing enzyme GAD. Both drugs were diluted in physiological saline (SAL, 0.9\%) shortly before use. Semicarbazide was injected at 5 or $7.5 \mu \mathrm{g} / 0.2 \mu \mathrm{L}$ and muscimol was injected at 1 or 2 $\mathrm{nmol} / 0.2 \mu \mathrm{L}$ into the dPAG or IC, 10 minutes before test sessions. The doses and time of injection were selected based on the results of previous studies from our laboratory (Castilho \& Brandão, 2001; Martinez et al., 2006; Reimer, Oliveira, \& Brandão, 2008).

\section{Microinjections}

The injection needle was a thin dental needle $(0.3$ $\mathrm{mm}$, o.d.) connected to a $10 \mu \mathrm{L}$ Hamilton syringe by means of a polyethylene tube (PE-10; BectonDickinson, NJ, USA). The injection needle was introduced through the guide-cannula until its lower end was $1 \mathrm{~mm}$ below it. The solutions were injected into the dPAG or IC with the help of an infusion pump (Harvard Apparatus, MA, USA). The displacement of an air bubble inside the polyethylene catheter connecting the syringe needle to the intracerebral needle was used to monitor the microinjection. The duration of microinjection was 1 minute and the needle was held in place for an additional minute to maximize diffusion away from the tip of the needle.

\section{Aversive Thresholds and Post-Stimulation Freezing}

The unconditioned test was conducted in a circular arena of transparent acrylic, measuring $60 \mathrm{~cm}$ in diameter and $50 \mathrm{~cm}$ high, with the floor divided into 12 sessions. The animals were allowed a 5 minute period of habituation in the arena at the beginning of each session. Afterwards, the brain was electrically stimulated using a sine wave stimulator (DV \& M, SP, Brazil) and the generated stimulation current was monitored by measuring the voltage drop across a $1 \mathrm{~K} \Omega$ resistor using an oscilloscope (Minipa, SP, Brazil). Brain stimulation $(\mathrm{AC}, 60 \mathrm{~Hz}, 10 \mathrm{~s})$ was presented at 1 minute intervals with the current intensity increasing by steps of $5 \mu \mathrm{A}$ for measurements of the aversive thresholds. Freezing threshold determined by the electrical stimulation of the dPAG or IC was operationally defined as the lowest intensity producing absence of any movement, except those related to respiration. The intensity of current producing running or jumping was considered to be the escape threshold. Animals with an escape threshold above $180 \mu \mathrm{A}$ (peak-to-peak) were removed from the experiment. In order to investigate the freezing behavior that persisted after interruption of $\mathrm{dPAG}$ and IC stimulation applied at the escape threshold, the animals remained in the arena for another $5 \mathrm{~min}$, without any stimulation, during which the freezing behavior was recorded. Soon after the measurement of these baseline values, rats received drug treatment into $\mathrm{dPAG}$ or IC and, 10 minutes later, the defensive behaviors were again evaluated through the measurements of the aversive thresholds and post-stimulation freezing.

\section{Histology}

Upon completion of the experiments, the animals were given a lethal dose of chloral hydrate $(500 \mathrm{mg} / \mathrm{Kg}$, i.p.) and $0.2 \mu \mathrm{L}$ of Evans Blue (2\%) were microinjected into the dPAG or IC in order to mark the position of the microinjection sites. Afterwards, rats were perfused transcardially with $0.9 \%$ saline followed by $4 \%$ of paraformaldehyde solution. Brains were removed from the skulls and maintained in paraformaldehyde solution for $2 \mathrm{~h}$. After this removal and storage process, they were cryoprotected in $30 \%$ sucrose until soaked. Serial $60 \mu \mathrm{m}$ brain coronal sections were cut using a freezing microtome and mounted on gelatin-coated slides and stained with cresyl violet (5\%) using Nissl protocol (Sigma-Aldrich, USA) in order to localize the positions of the microinjection sites according to the atlas of Paxinos and Watson (2007).

\section{Analysis of Results}

Data are presented as mean + S.E.M. Twoway analysis of variance (ANOVA) with repeated measures was used to assess the effects of muscimol or semicarbazide injections into the IC or $\mathrm{dPAG}$ on the aversive thresholds with treatments (drug $\mathrm{x}$ control) as between-subjects factor and conditions (freezing $\mathrm{x}$ escape) as within-subjects factor. Two-way ANOVA with repeated measures was also used for post-stimulation freezing analysis with treatments (drug x control) as between-subjects factor and conditions (baseline $\mathrm{x}$ test) as within-subjects factor. Significant comparisons were tested with Newman-Keuls test. Results of statistical tests with $\mathrm{p}$ lower than .05 were considered significant.

\section{Results}

The injection sites of all animals used in this study were located inside the dPAG or IC. Representative photomicrographs of the locations of the chemitrodes sites depicted on diagrams modified of the atlas of Paxinos and Watson (2007) into dPAG or IC are shown in Figure 1.

\section{Dorsal periaqueductal gray}

Figure 2A shows the mean change in the freezing and escape thresholds, determined by electrical stimulation of the dPAG across the baseline and test phases of the experiment in groups of animals injected with saline or muscimol (1 and 2 nmol) into dPAG. Two-way ANOVA with repeated measurements showed significant effect of treatments in the aversive thresholds $[F(2,39)=10.27, \mathrm{p}<.05]$. There were no 

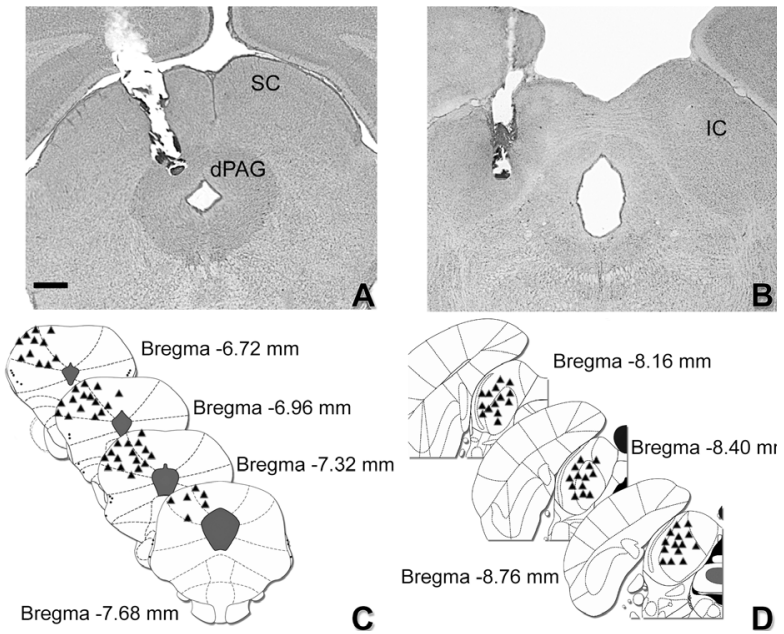

Figure 1. Photomicrograph showing representative sites of injections into the dorsal periaqueductal gray (A) and inferior colliculus (B). Outline of the injection sites in the dorsal periaqueductal gray and inferior colliculus on crosssections from the Paxinos and Watson atlas (2007) (C-D). The number of points in the figure is less than the total number of rats used because of several overlaps. $\mathrm{dPAG}=$ dorsal periaqueductal gray. $\mathrm{IC}=$ inferior colliculus. $\mathrm{SC}=$ superior colliculus. Scale bar equal to $0.5 \mathrm{~mm}$.
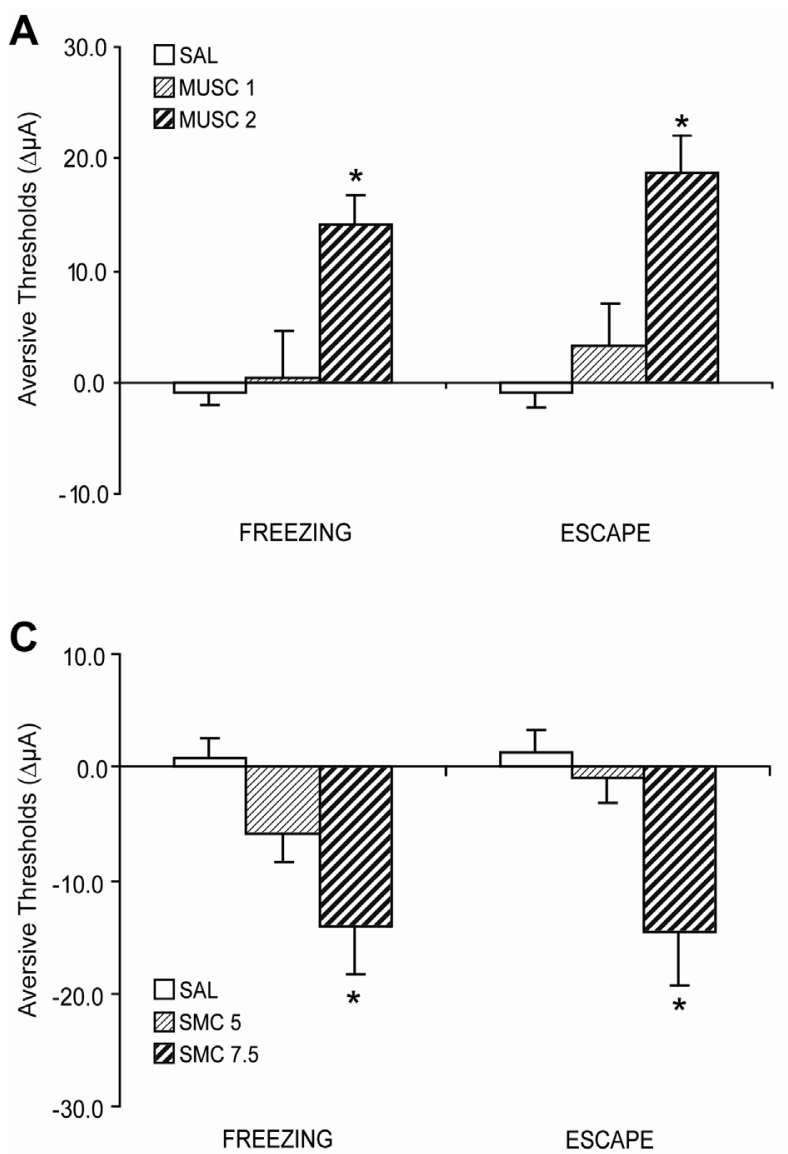

significant effects for conditions $[\mathrm{F}(1,39)=3.06, \mathrm{p}>$ $.05]$ or interaction between treatments vs. conditions $[\mathrm{F}(2,83)=0.83, \mathrm{p}>.05]$. Post-hoc comparisons using the Newman-Keuls test revealed increased freezing and escape thresholds in animals that received 2 nmol of muscimol when compared with control $(\mathrm{p}<$ $.05)$. Two-way ANOVA with repeated measures was also performed on the time of freezing following dPAG stimulation (Figure 2B). This analysis showed significant effect of conditions $[\mathrm{F}(1,39)=5.48, \mathrm{p}<.05]$. There were no significant effects for treatments $[F(2,39)$ $=0.07, \mathrm{p}>.05]$ or interaction between treatments vs. conditions $[\mathrm{F}(2,83)=1.13, \mathrm{p}>.05]$. Post-hoc comparisons using the Newman-Keuls test revealed significant differences in the post-stimulation freezing across baseline and test phases of the experiment ( $p$ $<.05$ ). Figure $2 \mathrm{C}$ shows the aversive thresholds, determined by electrical stimulation of the $\mathrm{APAG}$, in groups of animals injected with saline or semicarbazide $(5$ and $7.5 \mu \mathrm{g}$ ) into this same structure. Two-way ANOVA with repeated measures showed significant effect of treatments in the aversive thresholds $[\mathrm{F}(2,33)$ $=7.07, p<.05]$. There were no significant effects for
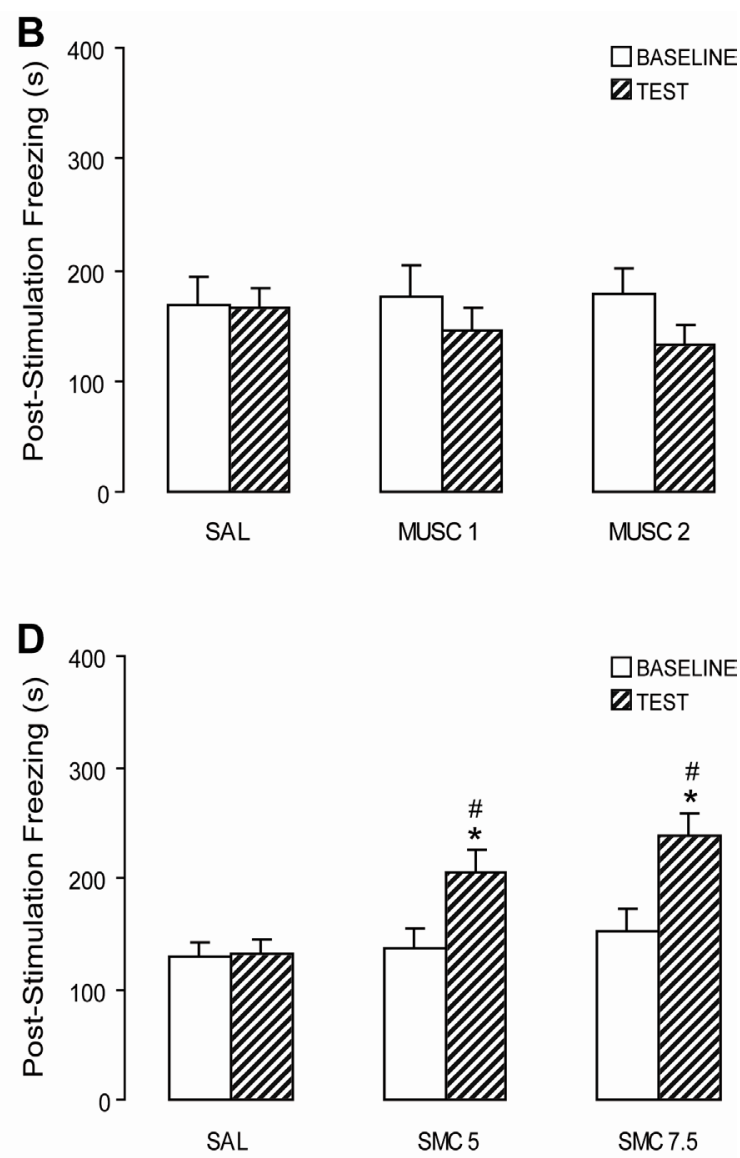

Figure 2. Differences $(\Delta)$ in thresholds of freezing and escape determined before and after intra-dPAG injection of saline, muscimol 1 or $2 \mathrm{nmol}(\mathrm{n}=12,15$ and 15 respectively) (A); or saline, semicarbazide 5 or $7.5 \mu \mathrm{g}(\mathrm{n}=12$ for all groups) (C). Mean $(+\mathrm{SEM})$ of time of post-stimulation freezing in groups of rats that received intra-dPAG injections of saline, muscimol $1 \mathrm{or} 2 \mathrm{nmol}$ ( $\mathrm{n}=12,15$ and 15 respectively) (B); or saline, semicarbazide 5 or $7.5 \mu \mathrm{g}(\mathrm{n}=12$ for all groups) (D). * $\mathrm{p}<.05$ in relation to the corresponding value in the saline group. $\# \mathrm{p}<.05$ in relation to its respective baseline. 

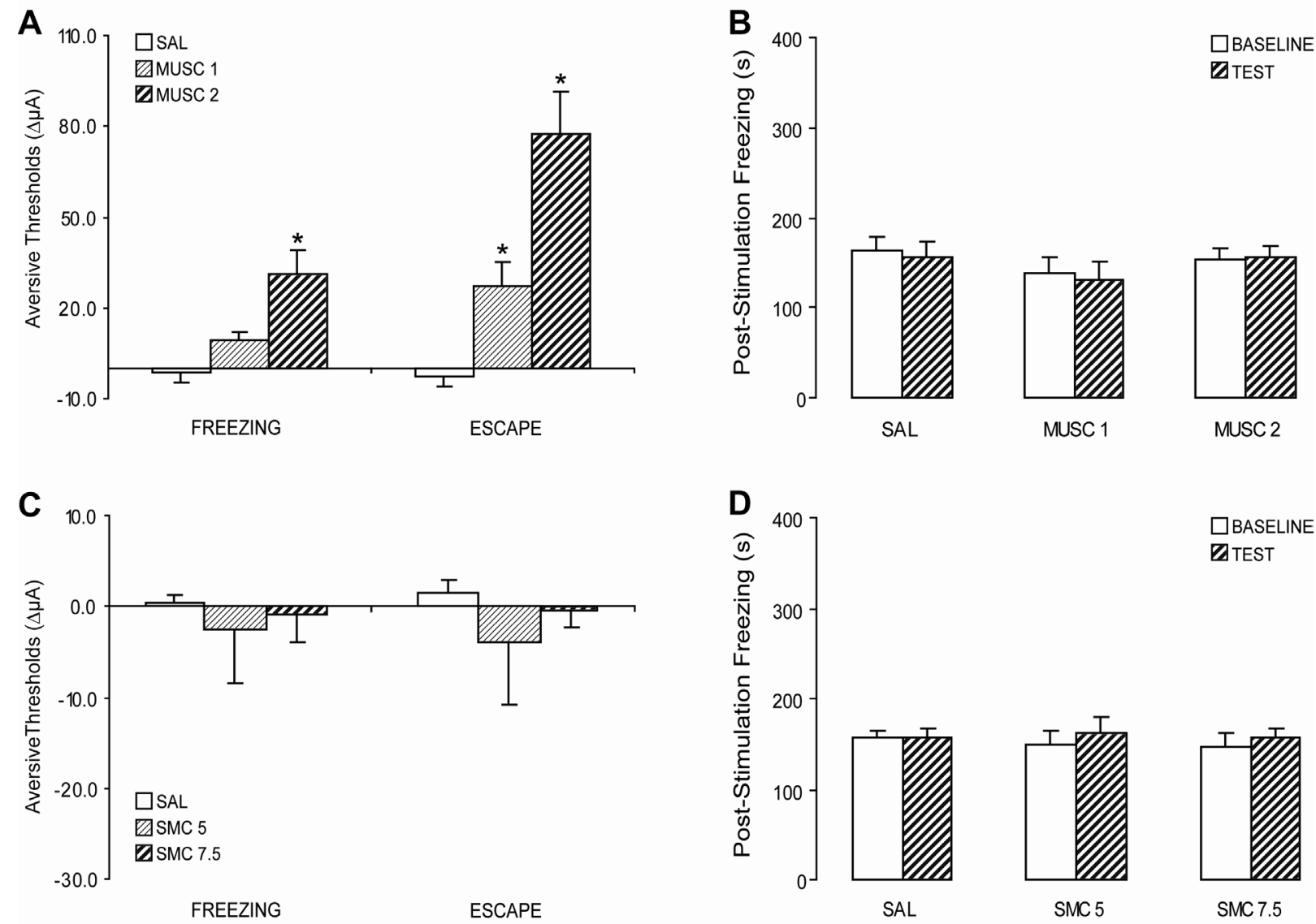

Figure 3. Differences $(\Delta)$ in thresholds of freezing and escape determined before and after intra-IC injection of saline, muscimol 1 or $2 \mathrm{nmol}$ ( $\mathrm{n}=9$ for all groups) (A); or saline, semicarbazide 5 or $7.5 \mu \mathrm{g}(\mathrm{n}=12$ for all groups) (C). Mean (+SEM) of time of poststimulation freezing in groups of rats that received intra-IC injection of saline, muscimol 1 or $2 \mathrm{nmol}$ ( $\mathrm{n}=9$ for all groups) (B); or saline, semicarbazide 5 or $7.5 \mu \mathrm{g}\left(\mathrm{n}=10,8\right.$ and 10 respectively) (D). ${ }^{*} \mathrm{p}<.05$ in relation to the corresponding value in the saline group.

conditions $[\mathrm{F}(1,33)=2.45, \mathrm{p}>.05]$ or interaction between treatments vs. conditions $[\mathrm{F}(2,71)=2.50, \mathrm{p}$ $>$.05]. Post-hoc comparisons with Newman-Keuls test revealed that $7.5 \mu \mathrm{g}$ of semicarbazide attenuated freezing and escape thresholds when compared with control $(\mathrm{p}<.05)$. Two-way ANOVA with repeated measures was also performed on the time of freezing following dPAG stimulation (Figure 2D). This analysis showed significant effect of treatments $[\mathrm{F}(2,33)=4.87$, $\mathrm{p}<.05]$, conditions $[\mathrm{F}(1,33)=28.60, \mathrm{p}<.05]$ and interaction between treatments vs. conditions $[\mathrm{F}(2,71)$ $=6.92, \mathrm{p}<.05]$. Post-hoc comparisons with NewmanKeuls test revealed that semicarbazide ( 5 and $7.5 \mu \mathrm{g}$ ) enhanced the post-stimulation freezing when compared to baseline and with the control group $(\mathrm{p}<.05)$.

\section{Inferior colliculus}

Figure $3 \mathrm{~A}$ shows the mean change in the freezing and escape thresholds, determined by electrical stimulation of the IC across baseline and test phases of the experiment, in groups of animals injected with saline or muscimol ( 1 and $2 \mathrm{nmol}$ ) into IC. Two-way ANOVA with repeated measures showed significant effects of treatments $[\mathrm{F}(2,24)=17.80, \mathrm{p}<.05]$, conditions $[\mathrm{F}(1,24)=22.77, \mathrm{p}<.05]$ and interaction between treatments vs. conditions $[\mathrm{F}(2,53)=10.18$, $\mathrm{p}<.05]$. Post-hoc comparisons with Newman-Keuls test revealed that muscimol ( 1 and $2 \mathrm{nmol}$ ) enhanced freezing and escape thresholds when compared with control $(\mathrm{p}<.05)$. Two-way ANOVA with repeated measures was also performed on the time of freezing following IC stimulation (Figure 3B). This analysis showed no significant effects of treatments $[\mathrm{F}(2,24)$ $=0.80, \mathrm{p}>.05]$, conditions $[\mathrm{F}(1,24)=0.22, \mathrm{p}>.05]$ or interaction between treatments vs. conditions $[F(2,53)=0.18, p>.05]$. Figure $3 \mathrm{C}$ shows the aversive thresholds, determined by electrical stimulation of the IC in groups of animals injected with saline or semicarbazide (5 and $7.5 \mu \mathrm{g}$ ) into this same structure. Two-way ANOVA with repeated measures showed no significant effects of treatments $[\mathrm{F}(2,27)$ $=0.26, \mathrm{p}>.05]$, conditions $[\mathrm{F}(1,27)=0.10, \mathrm{p}>.05]$ or interaction between treatments vs. conditions $[F(2,59)=0.74, p>.05]$. Two-way ANOVA with repeated measures was also performed on the time of freezing following IC stimulation (Figure 3D). This analysis showed no significant effects of treatments $[F(2,25)=0.06, \mathrm{p}>.05]$, conditions $[\mathrm{F}(1,25)=$ $4.11, \mathrm{p}>.05]$ or interaction between treatments vs. conditions $[\mathrm{F}(2,55)=0.87, \mathrm{p}>.05]$. 


\section{Discussion}

The disclosure of the mode a given brain structure participates when producing aspecific emotionalbehavior may contribute to our knowledge on the functioning of the neural circuitries underlying anxiety disorders. This study is an attempt to understand how GABA-A mechanisms of the dPAG and IC might modulate fear and anxiety-like states. To this end, we examined the functional influence of GABAergic mechanisms of dPAG and IC in freezing and escape responses elicited by aversive stimulation of these same structures and in the post-stimulation freezing. Although the use of the electrical brain stimulation has the disadvantage of activating both cell bodies and fibers of passage, this technique was selected due to the necessity for a gradual and discrete stimulation of these regions. Therefore, it was possible to observe the animal's behavior during the presence and the absence of the gradual increase of electrical stimulation.

As the intensity of the current applied to the dPAG was increased, the animals suddenly stopped, became immobile and often urinated and defecated. With higher intensities, freezing behavior was followed by vigorous running and jumping. The interruption of the electrical stimulation of dPAG at the escape threshold induced a long-lasting freezing behavior, the post-stimulation freezing.

Intra-dPAG injection of muscimol (2 $\mathrm{nmol})$ increased the freezing and escape thresholds determined by electrical stimulation of the dPAG. On the other hand, $7.5 \mu \mathrm{g}$ of semicarbazide reduced the freezing and escape thresholds when compared with control. Thus, while the activation of GABA-A receptors into $\mathrm{APAG}$ produced anti-aversive effects, attenuating the aversiveness of the electrical stimulation of this structure, the inhibition of GABA synthesis had opposite effects. These data are in agreement with previous studies showing an inhibitory role of the GABAergic transmission on the neural substrates of aversion in the dPAG (Aguiar \& Brandão, 1994; Audi \& Graeff, 1987; Bovier, Broekkamp, \& Lloyd, 1982; Brandão, Aguiar, \& Graeff, 1982; Di Scala \& Sandner, 1989; Motta \& Brandão, 1993). Semicarbazide ( 5 and $7.5 \mu \mathrm{g}$ ) also induced pro-aversive effects enhancing the dPAG post-stimulation freezing when compared to baseline and with the control group. Muscimol, however, did not change the time of freezing following dPAG stimulation. This result has to be interpreted with caution since a significant difference across baseline and test phases of this experiment could be observed. Probably, a higher dose of muscimol would be able to decrease dPAG post-stimulation freezing; so it should be used in other studies on this topic.

Overall, this paper presents further evidence for the important modulatory control exerted by GABAergic mechanisms of the dPAG in the expression of unconditioned defensive behaviors. Furthermore, this study also introduces new data, indicating that
GABA neurotransmission is involved in dPAG poststimulation freezing. This response has been related to the transference of aversive information passing through the dPAG to higher structures that are highly likely to process this kind of information, such as the amygdala. Likely, the transference of this information to the amygdala takes place through the laterodorsal nucleus of the thalamus. Indeed, as thoroughly described below, electrical or chemical stimulation of the dPAG caused substantial Fos labeling in this thalamic nucleus (Borelli, Ferreira-Netto, Coimbra, \& Brandão, 2005; Ferreira-Netto, Borelli, \& Brandão, 2005).

Despite their overt similarity, dPAG-evoked freezing and the dPAG post-stimulation freezing seem to be related to distinct behavioral defensive systems and model different anxiety disorders, panic attacks and panic disorder respectively. The initial freezing posture triggered by dPAG electrical stimulation seems to be a preparatory response for flight. The post-dPAG stimulation freezing persists at high levels after the stimulation and is not context dependent, suggesting that it is also unconditioned in nature (Vianna et al., 2001a, 2001b; Vianna\& Brandão, 2003). Ithas been proposed that freezing behavior allows the animals to acquire aversive information from the environment (Ferreira-Netto et al., 2005). So, a possible neural circuitry responsible for the occurrence of dPAG post-stimulation freezing might be through ascending projections to forebrain structures involved in the sensorial recognition of aversive stimuli. Indeed, stimulation of the dPAG at intensities causing freezing behavior causes simultaneous Fos-labeling of the laterodorsal nucleus of the thalamus, indicating that this structure is also involved in the sensorimotor gating activated by emotional stimuli at this midbrain level (Borelli et al., 2005; Ferreira-Netto et al., 2005). In a current study we are now investigating the involvement of GABAergic mechanisms of the laterodorsal nucleus of the thalamus in the dPAG post-stimulation freezing.

Similar to what happened for dPAG, gradual increases in the electrical stimulation of IC caused arousal followed by a period of immobility. As a result, the rats often urinated and defecated. With higher intensities, freezing behavior was followed by a state of behavioral activation expressed by running and jumping. Both running and jumping behaviors were more coordinated when the animals were stimulated in the inferior colliculus than when they were stimulated in the dPAG at the escape threshold (Brandão, Cardoso, Melo, Motta, \& Coimbra, 1994; Brandão et al., 1999).

Intra-IC injection of muscimol augmented the freezing and escape thresholds determined by electrical stimulation of the IC. Semicarbazide, however, had no effects in both aversive thresholds. Thus, while the activation of GABA-A receptors into IC attenuated the aversiveness of the electrical stimulation of this structure, the inhibition of GABA synthesis had no 
effect. The anti-aversive action of muscimol applied locally into the IC on the electrical stimulation of this structure had already been shown by other studies from this laboratory (Melo, Cardoso, \& Brandão, 1992; Pandossio \& Brandão, 1999). Despite the fact that earlier studies clearly demonstrated that the administration of GABAergic antagonists into the IC leads to the production of defensive behaviors (see Brandão et al., 1988, 1999, 2005), in the present study a decrease of GABAergic mediation with semicarbazide was unable to change the aversiveness of the electrical stimulation of this structure. Since both effects, that of microinjections of semicarbazide and that of electrical stimulation of the midbrain tectum, are probably due to the removal of the inhibitory control exerted by GABA on the defensive neural circuitry, a likely explanation would be that the electrical stimulation of the IC already promotes a significant reduction in GABAergic transmission, beyond which the semicarbazide no longer acts. Additionally, the difference between our results of microinjections of semicarbazide in $\mathrm{dPAG}$ and IC seems to depend on the particular structure under study so that the neural substrates of fear might subserve different aspects of the defensive reaction.

Like the dPAG, the interruption of the electrical stimulation of IC at the escape threshold also produced freezing (Martinez et al., 2006, 2007; Vianna \& Brandão, 2003). Muscimol and semicarbazide did not affect the time of freezing following IC stimulation. Thus, differently from the dPAG, GABAergic mechanisms therein do not appear to be involved in this response. As the inferior colliculus is primarily involved in the processing of auditory information it may be important in processing acoustic information of aversive nature as well. In fact, it has been proposed that neurons in the inferior colliculus serve as filters for sounds that require immediate action, such as certain sounds made by prey, predators and conspecifics (Brandão, Troncoso, De Souza Silva, \& Huston, 2003; Brandão et al., 2005). Given the differential involvement of the IC and APAG in the generation and integration of defensive behaviors more studies are still needed in order to better characterize the functional role of the IC post-stimulation freezing.

In summary, the current findings suggest that GABAergic mechanisms are important regulators of the expression of unconditioned fear in $\mathrm{APAG}$ and IC. However, only in dPAG GABAergic mechanisms appear to play a role on the sensory gating towards aversive information during post-stimulation freezing.

\section{Acknowledgements}

This research was supported by FAPESP (Proc. N. 06/06354-5) and CNPq (Proc. N. 472029/2006-0). A.R. Oliveira holds a Doctor scholarship from FAPESP and A.E. Reimer holds a Doctor scholarship from CNPq.

\section{References}

Aguiar, M.S., \& Brandão, M.L. (1994). Conditioned place aversion produced by microinjections of substance $\mathrm{P}$ into the periaqueductal gray of rats. Behavioral Pharmacology, 5, 369-373.

Audi, E.A., \& Graeff, F.G. (1987). GABAA receptors in the midbrain central grey mediate the antiaversive action of GABA. European Journal of Pharmacology, 135, 225-229.

Borelli, K.G., Ferreira-Netto, C., Coimbra, N.C., \& Brandão, M.L. (2005). Fos-like immunoreactivity in the brain associated with freezing or escape induced by inhibition of either glutamic acid decarboxylase or GABAA receptors in the dorsal periaqueductal gray. Brain Research, 1051, 100-111.

Bovier, P., Broekkamp, C.L., \& Lloyd, K.G. (1982). Enhancing GABAergic transmission reverses the aversive state in rats induced by electrical stimulation of the periaqueductal grey region. Brain Research, 248, 313-320.

Brandão, M.L., Aguiar, J.C., \& Graeff, F.G. (1982). GABA mediation of the anti-aversive action of minor tranquilizers. Pharmacology, Biochemistry and Behavior, 16, 397-402.

Brandão, M.L., Anseloni, V.Z., Pandóssio, J.E., De Araújo, J.E., \& Castilho, V.M. (1999). Neurochemical mechanisms of the defensive behavior in the dorsal midbrain. Neuroscience and Biobehavioral Reviews, 23, 863-875.

Brandão, M.L., Borelli, K.G., Nobre, M.J., Santos, J.M., AlbrechetSouza, L., Oliveira, A.R., \& Martinez, R.C. (2005). Gabaergic regulation of the neural organization of fear in the midbrain tectum. Neuroscience and Biobehavioral Reviews, 29, 1299-1311.

Brandão, M.L., Cardoso, S.H., Melo, L.L., Motta, V., \& Coimbra, N.C. (1994). Neural substrate of defensive behavior in the midbrain tectum. Neuroscience and Biobehavioral Reviews, 18, 339-346.

Brandão, M.L., Melo, L.L., \& Cardoso, S.H. (1993). Mechanisms of defense in the inferior colliculus. Behavioral Brain Research, $58,49-55$.

Brandão, M.L., Tomaz, C., Leão-Borges, P.C., Coimbra, N.C., \& Bagri, A. (1988). Defense reaction induced by microinjections of bicuculline into the inferior colliculus. Physiology and Behavior, 44, 361-365.

Brandão, M.L., Troncoso, A.C., De Souza Silva, M.A., \& Huston J.P. (2003). The relevance of neuronal substrates of defense in the midbrain tectum to anxiety and stress: Empirical and conceptual considerations. European Journal of Pharmacology, 463, 225-233.

Brandão, M.L., Zanoveli, J.M., Ruiz-Martinez, R.C., Oliveira L.C., \& Landeira-Fernandez, J. (2008). Different patterns of freezing behavior organized in the periaqueductal gray of rats: Association with different types of anxiety. Behavioral Brain Research, 188, 1-13.

Castilho, V.M., \& Brandão, M.L. (2001). Conditioned antinociception and freezing using electrical stimulation of the dorsal periaqueductal gray or inferior colliculus as unconditioned stimulus are differentially regulated by 5 -HT2A receptors in rats. Psychopharmacology, 155, 154-162.

Cuadra, G., Zurita, A., Macedo, C.E., Molina, V.A., \& Brandão, M.L. (2000). Electrical stimulation of the midbrain tectum enhances dopamine release in the frontal cortex. Brain Research Bulletin, 52, 413-418.

Di Scala, G., \& Sandner, G. (1989). Conditioned place aversion produced by microinjections of semicarbazide into the periaqueductal gray of the rat. Brain Research, 483, 91-97.

Ferreira-Netto, C., Borelli, K.G., \& Brandão, M.L. (2005). Neural segregation of Fos-protein distribution in the brain following freezing and escape behaviors induced by injections of either glutamate or NMDA into the dorsal periaqueductal gray of rats. Brain Research, 1031, 151-163.

Graeff, F.G. (1994). Neuroanatomy and neurotransmitter regulation of defensive behaviors and related emotions in mammals. Brazilian Journal of Medical and Biological Research, 27, 811-829.

Graeff, F.G. (2002). On serotonin and experimental anxiety. Psychopharmacology, 163, 467-476.

Graeff, F.G. (2004). Serotonin, the periaqueductal gray and panic. Neuroscience and Biobehavioral Reviews, 28, 239-259.

Graeff, F.G., \& Del-Ben, C.M. (2008). Neurobiology of panic disorder: From animal models to brain neuroimaging. Neuroscience and Biobehavioral Reviews, 32, 1326-1335.

Graeff, F.G., Silveira, M.C., Nogueira, R.L., Audi, E.A., \& Oliveira, R.M. (1993). Role of the amygdala and periaqueductal gray in anxiety and panic. Behavioral Brain Research, 58, 123-131.

Martinez, R.C.R., Oliveira, A.R., \& Brandão, M.L. (2006). Conditioned 
and unconditioned fear organized in the periaqueductal gray are differentially sensitive to injections of muscimol into amygdaloid nuclei. Neurobiology of Learning and Memory, 85, 58-65.

Martinez, R.C.R., Oliveira, A.R., \& Brandão, M.L. (2007). Serotonergic mechanisms in the basolateral amygdala differentially regulate the conditioned and unconditioned fear organized in the periaqueductal gray. European Neuropsychopharmacology, 17, 717-724.

Melo, L.L., Cardoso, S.H., \& Brandão, M.L. (1992). Antiaversive action of benzodiazepines on escape behavior induced by electrical stimulation of the inferior colliculus. Physiology and Behavior, 51, 557-562.

Motta, V., \& Brandão, M.L. (1993). Aversive and antiaversive effects of morphine in the dorsal periaqueductal gray of rats submitted to the elevated plus-maze test. Pharmacology, Biochemistry and Behavior, 44, 119-125.

Pandossio, J.E., \& Brandão, M.L. (1999). Defensive reactions are counteracted by microinjections of midazolam and muscimol and elicited by activation of glutamate receptors into the inferior colliculus. Psychopharmacology, 142, 360-368.

Paxinos, G., \& Watson, C. (2007). The rat brain in stereotaxic coordinates. San Diego: Academic Press.

Reimer, A.E., Oliveira, A.R., \& Brandão, M.L. (2008). Selective involvement of GABAergic mechanisms of the dorsal periaqueductal gray and inferior colliculus on the memory of the contextual fear as assessed by the fear potentiated startle test. Brain Research Bulletin, 76, 545-550.

Thompson, S.G., \& McGeer, E.G. (1985). GABA-transaminase and glutamic acid decarboxylase changes in the brain of rats treated with pyrithiamine. Neurochemical Research, 10, 1653-1660.

Troncoso, A.C., Osaki, M.Y., Masson, S., Borelli, K.G., \& Brandão, M.L. (2003). Apomorphine enhances conditioned responses induced by aversive stimulation of the inferior colliculus. Neuropsychopharmacology, 28, 284-291.

Vianna, D.M., \& Brandão, M.L. (2003). Anatomical connections of the periaqueductal gray: Specific neural substrates for different kinds of fear. Brazilian Journal of Medical and Biological Research, 36, 557-566.

Vianna, D.M., Graeff, F.G., Brandão, M.L., \& Landeira-Fernandez J. (2001a). Defensive freezing evoked by electrical stimulation of the periaqueductal gray: Comparison between dorsolateral and ventrolateral regions. Neuroreport, 12, 4109-4112.

Vianna, D.M., Landeira-Fernandez, J., \& Brandão. M.L. (2001b). Dorsolateral and ventral regions of the periaqueductal gray matter are involved in distinct types of fear. Neuroscience and Biobehavioral Reviews, 25, 711-719 